\title{
Ontologically-systematized decision-making and intellectual environment of engineering interaction
}

\author{
Valentina Goryunova \\ Penza State Technological University \\ Penza, Russia \\ gvv17@ya.ru
}

\begin{abstract}
The article presents ontologically systematized means of creating an intellectual environment for engineering interaction in the context of production system. It is noted that the use of modular ontologies to ensure data stability is a key factor in the "cross-linking" of local subject areas in integrated systems for planning production resources. The composition of ontological modules, which should be presented in an intelligent engineering interaction environment, is considered in the form of targeted knowledge about tasks and methods for their solution within the framework of the modeled domain. A method of decision support for the management of production systems is proposed, implemented using modular and ontological technology in Petri nets and rules.
\end{abstract}

Keywords - ontology, management, intellectual environment of engineering interaction; technology of the ontological system, Petri nets, production systems

\section{INTRODUCTION}

Studies of production systems are one of the important areas of application of the general theory of systems. The most important role in managing the design and operation is the use of integrated information environments that perform the modeling of organizational and technological processes[1].

The solution of these and other problems of modeling and analysis of organizational processes in modern conditions involves the use of integrated environment of engineering interaction, using the decomposition of the processes of organizational and technological management[2]. And the application of ontology engineering tools, allowing to form preliminary guidelines for the project management system, allows you to identify and eliminate inconsistencies and errors at the earliest possible stages of its implementation.

We will be interested in various issues related to the interaction of subsystems, the connection of which forms the system as a whole, and the research objective will be the development of the intellectual engineering interaction environment (IEEI) used by the production. IEEI is viewed as a family of individually perceived and interconnected decisionmaking subsystems

Subsystems (modules) form the basis of the integrated engineering interaction environment [3].

Ontological system technology defines the mechanism of designing, functioning and development of the integrated engineering interaction environment (standard version, hierarchical type) from the so-called declarative ontological modules. At the same time, the static structure of a distributed network of ontological modules determines the "strategy" of decision-making processes [4], and the dynamic mechanism of "learning" processes describes an "alternative" to operational processes in ontological blocks (OB). As a formal device, Petri nets and production model.

\section{ONTOLOGY AND INTEGRATED ENVIRONMENT OF ENGINEERING INTERACTION}

The main decisions that determine the enterprise are called architecture. But components, modules, placements (and various other objects) of the enterprise are not so easy to determine - therefore, it is necessary to move from an architectural description to an ontological [5].

One of the founding fathers of architectural modeling of enterprises was John Zachman. Ontologically, the Zachman enterprise consists of the following objects:

1. Assets (inventory sets) - entities and relationships.

2. Process flows (process flows) - transformations, and that at the input / output of these transformations.

3. Distribution networks (distribution networks) - this is operational management, logistics networks, taxiing flows.

4. Responsibility / authorization (responsibility assignments) - this is the "organization".

5. Timing Cycles.

6. Motivation Intentions - goals and means, what tools help to achieve what goals.

All these objects are connected to each other via correspondence rules - as it is supposed in ISO 42010.

The knowledge bases of most integrated systems of largescale production contain specific knowledge from the subject area (subject knowledge) within individual ontological modules, which are represented as instances of concepts 
(specific objects) and relationships between them - instances of relationships or constraints specified on the values of attributes of instances of concepts. Each separate ontological module executes a stream modular script and is formed as a hierarchy of stream scenarios of processes consisting of operations.

The structure and properties of any system can be effectively investigated using the vocabulary of terms used to describe the characteristics of objects and processes relevant to the system under consideration, accurate and unambiguous definitions of all terms in this dictionary and the classification of logical relationships between these terms [6]. The set of these tools is the ontology of the system, it provides a structured methodology, through which it is possible to visually and efficiently develop, maintain and formalize the intellectual integration environment for managing product data. The design of intellectual environment of engineering interaction (IEEI) is decomposed into stages. At the same time, there are a number of features that determine the nature of the implementation of individual stages[7]. These features include: Collective use of knowledge, which involves the pooling and distribution of knowledge sources for different actors, and therefore, the solution of organizational issues of administration and optimization of business processes that connect individual users. The composition of knowledge sources is determined in principle, specific sources of knowledge, especially external sources of knowledge, can be added as the project develops. Because the intellectual information environment has a multi-purpose purpose, there is a need to integrate diverse sources of knowledge based on a single semantic description of the knowledge space. Thus, the conceptual elaboration of the implementation of IEEI is basically reduced to the creation of an ontology, which is performed classically as a result of interaction between performers and experts. The development and support of ontology in the life cycle of the product requires constant efforts to develop it. Since various types of activities are often involved in the process of manufacturing and selling a product, several ontologies may be required for such an intelligent information environment. In the formulation of the problem of using single complex means for describing the static and dynamic characteristics of the process of designing the production and operation of engineering products, the design ontology becomes the central task in developing an intelligent information environment [8].

Ontology is an exact (explicit) description of the conceptualization of knowledge in the form of a set of concepts (concepts) used and a set of paradigmatic and syntagmatic relations. Paradigmatic relations are the relations of synonymy, homonymy, polysemy, generalization "gender-kind", aggregation "whole-part", cause-effect and other relationships that turn the vocabulary of concepts (concepts, categories) into a thesaurus. Syntagmatic relations present semantic limitations in the form of axioms or logical rules that allow you to correctly build variable relations of concepts in a specific context of the solution of the problem.

Formalization of the semantic structure of knowledge requires the use of predicate calculus languages for mapping axiomatics and object-oriented representation of knowledge for mapping the taxonomy of the concepts used [8].
In these conditions, a special role is played by information management. The head (expert) interacts with the system of support for the adoption of information management decisions, which in an automated mode solves the following main tasks: assessment of the information environment, selection (formation) of goals, strategies, management impacts; Assessment of the effectiveness and modification of the process of achieving the goals, the success of the implementation of selected strategies, the effectiveness of impacts; Accounting for the manager's subjective preferences, etc. The analysis of the effectiveness of operational impacts, the correctness of the strategies implemented and the degree of success in achieving the goal can be conducted according to different criteria, but simultaneous (parallel) implementation of interconnection at all three levels of managerial decisions: operational impact - strategy - goals should be ensured [9].

The formation of targeted strategies is considered in a very large number of works. Among them, one should single out a series of monographs by $\mathrm{H}$. Mintzberg, in which ten directions are examined that examine the choice of the OTC strategy from different angles of view (schools of design, planning, positioning, entrepreneurship, training, power, culture, environment, configuration, cognitive school)

Within the framework of the ontology we will distinguish three sub graphs:

1. The T-graph is the conceptual part of the ontology. In this graph, the classes $\mathrm{T}$ and $\mathrm{R}$ are nodes, and the arcs are the base relations introduced by the structure of the organizational system.

2. A-graph is an object-oriented graph. Its nodes are a set of ontology object-targets, arcs are relations between objectstargets, as entered by the target structure, and the relations $\mathrm{R}$ introduced on the T-graph.

3. TA-graph - a link between the conceptual and the object. As the nodes contains the classes $\mathrm{T}$ and the objects $\mathrm{A}$ belonging to these classes, the relations of belonging to the class are used as arcs. This graph is bipartite.

$$
\text { Ont }=\mathrm{T}+\mathrm{A}+\mathrm{TA}
$$

The Petri net is a discrete model based on a labeled oriented graph that allows modeling asynchronism and parallelism of independent events, pipeline-type parallelism, complex synchronization forms, and conflict interactions between processes.

The ontological-systematized approach for intellectual support of management decisions, suggested in the article, assumes that the processes consist of purposeful actions operations performed in the order determined by the ontology model (Fig. 1).

The result of the simulation of the protocol is the optimal (according to the criteria of the effectiveness of the solutions and the level of attainability of the goals) the image of the protocol of the conceptual mask, based on the Petri network of a special type in the levels of managerial decisions (goalstrategic decision-operational impact) and the register of 
classification data for the modules (containing Ranks "activityaction-operation").

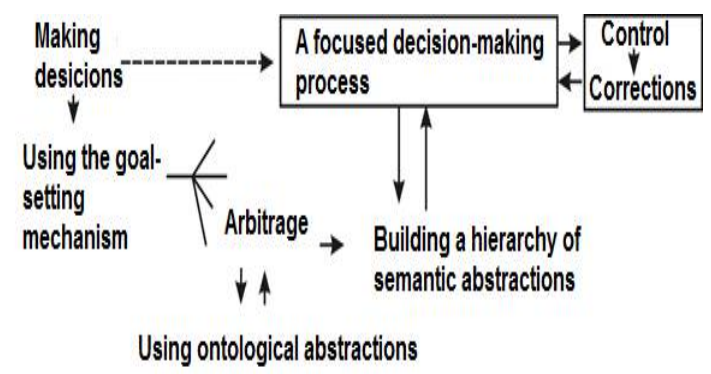

Fig. 1. Ontologically-systematized approach for intellectual support of management decisions

Typical modules for supporting solutions in production systems are:

1) Modeling of the enterprise. Facilitate the reduction of the timing of implementation;

2) Production module. Requirements planning, product configurator, serial production management;

3) Process module. Supports the production process from research and development to production, supply, sales;

4) Finance module. Financial accounting system;

5) Supplies and warehouses. Manages sales, purchases;

6) Project module. Designed to work projects and prepare a commercial proposal;

7) Module of the administrator of the enterprise. Toolkit for improving economic activity;

8) The module of transport. Transportation;

9) The service module. Management of all types of service.

Another important type of knowledge that you need to represent in IEEI is knowledge about the tasks and methods for solving them within the framework of the modeled subject area (methods and algorithms). This knowledge characterizes the problem area of the intellectual system. This kind of knowledge can be both declarative and procedural. The first type includes knowledge describing the space of solved IEEI problems, including the division of tasks into subtasks and the connection of subtasks with methods for their solution, to the second - knowledge representing both methods for solving problems, and specific algorithms. In addition to knowledge presented in the knowledge base, practically in any IEEI it is necessary to represent knowledge describing a fragment of reality (situation), which specifies the context and input data for tasks solved by IEEI. Such knowledge, as well as subject knowledge, are usually represented as instances of concepts and relationships and/or constraints defined on the values of the attributes of instances of concepts.

Based on the above requirements, the concept of an integrated knowledge representation model consisting of ontological blocks is proposed. The ontological block model combines various complementary means and methods of representing and processing knowledge of the following models: ontological, network, production, imperative.

All means of representation and processing of knowledge of the integrated model are combined within the framework of one ONT_OB ontological block formalism. This approach ensures the possibility of sharing all the means of integrated models, and, consequently, a joint description of declarative and procedural knowledge [10].

Based on the studies conducted within the framework of the conceptual-purpose approach, the ontology of the OS can be displayed in the form of a graph structure. Then let the ontology ONT be some graph

$$
\mathrm{ONT}=<\mathrm{N}, \mathrm{E}>\text {, }
$$

where $\mathrm{N}$ - nodes of ontology, $\mathrm{E}-\mathrm{E}$ - relations between nodes (oriented arcs).

Thus, MOST, defines a complex description of the concepts and relationships of simulated software in the form of classes of objects and relationships that encapsulate the semantic properties and constraints on their attributes and arguments. This determines the semantic and functional structure of the network, which appears to be a separate module of subject knowledge and knowledge about the task.

Formal definition $\operatorname{MOST}=<\mathrm{N}, \mathrm{S}, \mathrm{F}, \mathrm{a}, \mathrm{b}>$ ),

where $\mathrm{N}$ is a special type Petri net.

$\mathrm{N}=<\mathrm{P}, \mathrm{T}, \mathrm{I}, \mathrm{O}, \mathrm{M} 0)>$,

where $\mathrm{P}=\{\mathrm{pi} / \mathrm{i}=1-\mathrm{n}\}$ is the set of flow objects,

$\mathrm{T}=\{\mathrm{Ti} / \mathrm{i}=1-\mathrm{m}\}$ is the set of transition operations,

$\mathrm{I}=\mathrm{P} * \mathrm{~T}->\{0,1\}$ is the input function,

$\mathrm{O}=\mathrm{T} * \mathrm{PT}->\mid 0,1\}$ is the output function,

M0: $\mathrm{P}-\{0,1\}$ is the initial marking;

$\mathrm{S}=\{\mathrm{si} / \mathrm{i}=1-\mathrm{n}\}, \mathrm{S}$ is the set of information elements of the object represented by the tables. $\mathrm{S}$ is the mapping set to "load" items in the object position;

$\mathrm{F}:\{\mathrm{fi} / \mathrm{i}=1-\mathrm{n}\} ; \mathrm{F}$ is the set of logical formulas

a: $\mathrm{P}->\mathrm{S}$ is the mapping set to "load" items in the object position;

b: $\mathrm{T}->\mathrm{F}$ is the mapping that defines the "load" of the logical transition formulas.

The ontological block is an ontological structure of conceptual types of the subject domain (PR), specific for some conceptual relations and lexical means. The system integration of the specified ontologies (linguistic and subject area) together with the facts base and inference rules for the considered PR represents the KnowledgeBase, and the grammatical processor forms the structural units of the corresponding hierarchies.

IEEI consists of the following control units:- Information management unit in the enterprise;- block of hardware and technical base and means of communication;- DBMS block, system and providing software;- a block of software products that automate the management of information processes;- the 
block providing the rules for the use and development of software products;- IT department and providing services;block of users of software products.

Formally, the ONT_OB model can be represented by a six:

$$
\text { ONT_OB }=<\text { MOST, SN, FN, PS, IM, MD }>\text { where, }
$$

MOST is an applied ontology that defines the descriptions of the main entities (concepts and relations) of the object (PO) and problem areas (PR) of the application in the form of object classes and relations;

$\mathrm{SN}$ - is an object-oriented semantic network whose object properties and relations are defined by the MOST ontology.

FN - functional network, including constraint instances, set on the values of attributes of SN semantic network objects;

PS - is a system of product rules, described in terms of classes and MOST ontology relationships and operations on their instances;

\section{MD - means of imperative programming.}

IEEI implement processes that consist of separate, purposeful operations performed in a certain order. In this case, the elements of the IEEI of the $\mathrm{i}$-th level $(\mathrm{i}=0,1, \ldots, \mathrm{K}-1$, where $\mathrm{K}$ is the number of levels) implements processes based on the operations realized by the $\mathrm{i}+1$-level modules. In other words, in IEEI there is a hierarchy of processes, during which flow moves [6]. Processes interact with each other and with the external environment of the IEEI-system through flows (information, material, financial, etc.). Streams can correspond to mobile objects "passed through" the system (orders, products and their components, transported goods), and resources supporting the life of the IEEI system (raw materials, energy, human factor). Since object flows are discrete, for the uniformity of the model we assume that the resource flows are discrete, i.e. Resources are consumed in portions. A portion of the flow will be called its element.

The design of ontologically-systematized IEEI modules proceeds from the phases of complex system design adopted in the SADT methodology: 1) analysis - determining what the system will do; 2) design - definition of subsystems and their interaction; 3) implementation - the development of subsystems separately, combining - connecting subsystems into a single whole; 4) testing - checking the operation of the system; 5) installation - putting the system into operation; 6) functioning - using the system.

Ontological systems are designed to make knowledge a collective property of a wide range of people, to provide a powerful tool for fixing, acquiring and processing knowledge, checking them for consistency, completeness and so on. In addition, the knowledge representation for ontological modules is systematized. A glossary of terms is compiled and documented.

As a rule, the technique is reduced to an algorithm that is iterative in nature. For the development process, it is necessary to provide a number of "control points" to check the results obtained for compliance with the selected criteria. These criteria should be correlated with the given criteria for designing the knowledge base of the subject area, since the creation of the latter is the goal for developers [9]. The composition of the ontology-systematized analysis of the PR includes:

- a reasonable choice of the exact (and sufficient) fragment of the subject area for which the user's tasks will be placed and solved within the framework of the ontological module;

- choice of methods and procedures of system-ontological analysis, which, in particular, can be abstraction and specification, composition and decomposition, structuring, clustering and classification, testing and verification;

- compilation of a detailed vocabulary of terms and its subdivision into subsets of terms-objects, terms-processes and terms, which name tasks and methods.

The methodology of ontology design of the subject area involves the formation of sets of concepts, relationships, interpretation functions and axioms. Building these sets manually is a laborious process, both in time and in the number of highly skilled specialists involved in the design process. Ontologically-systematized approach to integration of modules of IEEI presupposes the creation of instrumental software systems [10].

Using an ontologically systematized approach, a comprehensive solution is proposed for the development of IIS, taking into account the criterial values of the indicators included in the ontological blocks (declarants).

Ontologically-systematized approach provides for three groups of tools. The first group includes tools for creating ontologies that involve supporting collaborative development and viewing, creating an ontology in accordance with this (arbitrary) methodology, supporting reasoning. The second group includes tools for combining, collating and harmonizing ontologies. The teaching involves looking for similarities and differences between the initial ontologies and creating a resulting ontology containing elements of the original ontologies. The matching procedure consists in finding the semantic links of different ontologies. The ontology alignment procedure establishes various types of correspondence between two ontologies, the information of which is stored for further use in user applications

\section{ONTOLOGICALLY-SYSTEMATIZED APPROACH TO DECISION-MAKING SUPPORT FOR LARGE-SCALE PRODUCTION}

Each purposeful element of a large-scale system must be analyzed in a special way. At the same time, multidimensional modeling of large-scale production involves the development of a formalism that allows modeling and researching the results of multi-problem analysis of the relevant subject area [11]. The multidimensional model is constructed as a formal language, intended for a multidimensional expression of the concepts of the subject domain. The main problem of the ontologicalsystematized approach is the creation, research and use of tools that allow to represent the framework of formal models perceived as a set of interrelated and complementary descriptions of the same subject area, leaving a single whole. In order to create such a framework it is necessary to use the 
methodology of multi-problem analysis and the technology of modular multidimensional modeling. Any methodology for the analysis of the subject domain should determine the techniques for identifying significant entities, methods for formalizing knowledge and the form of documenting the results. A formal theory is a set of theorems that is closed under the rules of inference. In practice, any theory is limited to its field of application, and even in this area, as a rule, is incomplete, i.e. generates along with excellent predictions and inadequate results. And an attempt to solve the problem of incompleteness by expanding the existing theory almost always leads to conceptual inconsistency. Every formal theory is defined by a formal language that generates formulas that make sense from the point of view of this theory, and a set of theorems interpretable in some domain as feasible. For a constructive construction of a formal theory, a finite alphabet is fixed, an enumerable set of formulas is determined, a solvable set of axioms is singled out, a finite set of inference rules are given, allowing one to obtain new formulas (theorems) on the basis of the available ones. With an ontologically systematized approach, for each active problematic, it is necessary to identify the entities of the subject domain that are correlated with certain concepts. Techniques for formalizing knowledge need to be realized by describing ways of expressing and interpreting concepts, and as a form of documentation, one should use conceptual structures in which ways of abstracting concepts are specified.

A conceptual structure is understood as the totality of concepts for which the methods of their formation (abstraction) are given. The bearer of the conceptual structure is a set of concepts, and its signature is the set of mappings of generalization, typing, aggregation and association. The abovementioned abstractions are sufficient for expressing any interrelation of the concepts of description on the basis of formalized knowledge. For each concept from a conceptual structure, its syntax and semantics are specified.

The syntax of a concept is described as one or more forms of its expression, and semantics as one or a set of named pragmatists defining the interpretations of each form of the concept expression in the text. The name of pragmatics defines an aspect within which each form of expression of concepts is interpreted.

To describe the semantics of a multidimensional model, the method of semantic induction is used, which consists of using semantic categories, which are defined as necessary, in the process of describing the syntax of a formal language and the means of that language.

The integration of conceptual structures, methods of expression and interpretation of concepts obtained ontologically systematized analysis of some subject area will be considered as its framework model consisting of declarative modules. Unlike other known formalisms, with such modeling, it becomes possible to express the accumulated knowledge about the subject area in the most natural form and to specify not one, but many interpretations for decision making alternatives. Moreover, in the description of the subject domain itself, it is possible to use various aspects when interpreting different fragments of the domain [12]. This allows us to use the formal means necessary to express the purposefulness of a large-scale system through the purposefulness of its elements.

The essence of the modular-ontological system technology lies in the fact that for each problematic it creates its own framework of formal descriptions of sets of rules, inherent only in this problematics, which combine to create a multi-problem description of the subject area.

For each active problematics, a conceptual analysis of the problem area is performed and its conceptual structure is found. The conceptual structures resulting from the ontological-systematized analysis unite and form the ontology of the subject domain.

The solution of the general problem of ontological system modeling is given in the form of a situational description - a collection of facts (judgments) that take place and a solution (properties of the solution) of a standing application in the form of expressions (conclusions) acceptable for the resulting final language.

\section{CONCLUSION}

In the methodology of the ontological-systematized approach, the preliminary focused analysis of the subject area and the compilation of a detailed technical design assignment is included. This stage (as well as the process of designing the knowledge base of the company as a whole) is of a complex analytical nature and consists in multiple abstraction. Knowledge of the subject area, understanding of the essence of the processes occurring in it, laws, rules and restrictions governing its development, is a necessary condition for the successful solution of problems solved in the conditions of large-scale production. Moreover, the availability of such knowledge is a necessary condition for the formulation, formulation of these tasks, without which the solution itself is impossible.

Thus, ontologically systematized methods for integrating IEEI modules are proposed. A method for modeling modules and business processes implemented using modular and ontological technology in Petri nets and rules is proposed.

\section{REFERENCES}

[1] Burkov V., Goubko M., Korgin N., Novikov D. Introduction to Theory of Control in Organizations. - New York: CRC Press, 2015, $352 \mathrm{p}$.

[2] New Frontiers in Information and Production Systems Modeling and Analysis - Incentive Mechanisms, Competence Management, Knowledge-based Production / Series "Intelligent Systems Reference Library". Ed. by P. Rozewsky, D. Novikov, O. Zaikin, N. Bakhtadze. - Berlin: Springer, 2016, 268 p.

[3] Novikov D., Chkhartishvili A. Reflexion and Control: Mathematical Models. - London: CRC Press, 2014, 298 p

[4] Goryunova V.V., Goryunova T.I., Zhilyaev PS Ispol'zovanie sredstv ontologicheskoj podderzhki $\mathrm{V}$ korporativnyh informacionnyh sistemah organizacionnogo upravleniya [Using ontological support in corporate information systems of organizational management]. In the collection: Theory of active systems Materials of the international scientific and practical conference. Under the general editorship of VN. Burkova. 2014, pp 241-244

[5] Management of the development of information systems Textbook for high schools / Edited by G.N. Kalyanova Vasiliev R.B., 
Kalyanov G.N., Lyovochkina G.A. 2017. Moscow. Hot lineTelecom $376 \mathrm{p}$.

[6] Gavrilova T., Leshcheva I. Ontology Design and Individual Cognitive Peculiarities: A Pilot Study // Expert Systems with Applications, available online DOI information: 10.1016/j.eswa.2015.01.008

[7] Novikov D. Control Methodology. - New York: Nova Science Publishers, 2013, 76 p.

[8] Goryunova V.V. Analysis of operational-technological processes with application of modular systematic ontology Bulletin of the Kazan State Technical University. A.N. Tupolev. 2010, № 1, pp. 97-102

[9] Goryunova V.V. Conceptual specifications of operationaltechnological processes. International Journal of Experimental Education. 2015, № 12-4, pp. 572-577
[10] Goryunova V.V., Proektirovanie sistem tekhnicheskogo obsluzhivaniya i remonta $\mathrm{s}$ ispol'zovaniem ontologij, [Design of maintenance and repair systems using ontologies] [Neurocomputers: development and application]. 2009, № 12, pp. 23-28.

[11] Goryunova V.V. Methods of intellectualization of solving the problems of logistic management of technical means of the Ministry of Emergency Situations. Technologies of technospheric security 2016, №1, pp. 150-153

[12] Lukinova O.V., Komp'yuternyj monitoring sostoyaniya sredy biznes-processov pri ehkspluatacii sistemy zashchity, [Computer monitoring of the environment of business processes during the operation of the protection system] [Open Education] №. 4, 2012, pp. 37-47. 